\title{
A Novel Model and GA-Based Solution for Resource Scheduling in Highway Emergency Rescue
}

\author{
Yuxin Niu \\ School of Highway, Chang'an University,xi'an,China \\ yunext1@126.com
}

\begin{abstract}
Keywords: Highway, Emergency Rescue, Vehicle Schedule, Genetic Algorithm Abstract. Highway is the major artery and main component of China's transportation system and plays a vital role in national economic construction. After an accident on the highway, how to quickly reach the accident point through effective dispatching and implementing emergency rescue operations is extremely important. Further, when the transportation conditions (such as traffic status) varies on different road segments at different time intervals, it is a very challenging problem to distinguish between priorities and properly dispatch emergency rescue resources. In this paper, it is taken as the key optimization goal to minimize the delay of emergency rescue response. And then, a genetic algorithm for solving the corresponding model is presented by adopting the random walk method. In our GA, a chromosome is represented by a transition matrix for defining the random walk, and specific crossover operation and mutation operation are given in details. The experiment on our GA is implemented by using GA-Toolbox of MATLAB, and the results manifest that our proposal is effective. Finally, the case of emergency rescue resource scheduling problem for the multiple accidents points is also discussed.
\end{abstract}

\section{Introduction}

China's highways have been invested and built since 1988, and thus entered the stage of gold development. By March 2017, the total mileage of China's highways has reached 125,373 kilometers [1], forming a highway network with a considerable scale due to its traffic volume. Larger, extremely high transport performance, its position in the road traffic and transportation industry is getting higher and higher. Under the background of the continuous expansion of the highway network, the demand for supporting facilities and services along the line has become more and more prominent. Therefore, the focus of the development of the highway has gradually shifted from construction to operation and management, and it has managed to realize the safety of the highway. Operation and smooth passage become the key. In 2013 alone, there were 8,693 highway accidents across the country, causing 5,843 deaths and 11,169 injuries. The casualty rate was as high as $34.34 \%$ [2]. The highway safety situation is grave.

After an accident on the highway, how to quickly reach the accident point through effective dispatch and implement emergency rescue operations is extremely important. The dispatch of emergency resources is faced with various complicated situations, such as how many accidents occur, the time of accidents, the degree of traffic jams at accidents, and the location of resource points. When there are multiple road accidents at the same time, it is also a very challenging problem to prioritize and dispatch emergency rescue resources and to choose a reasonable path if the priorities are different.

Fang [3] used the frog leaping algorithm to give the solution to the optimal path to a single accident point. Dong et al. [4] used genetic algorithms to study the dynamic path planning problem of the time-dependent dynamic network model. Fang et al. [5] proposed a corresponding emergency system model under the premise of meeting the time urgency of the emergency system. Sundar et al.[6] researched a path planning problem where a team of Unmanned Vehicles was required to visit a given set of targets, and developed a primal-dual heuristic and incorporate the heuristic in a Lagrangian relaxation procedure to find good, feasible solutions and lower bounds for the path planning problem.

In this paper, it is taken as the key optimization goal to minimize the delay of emergency rescue response. And then, the emergency rescue resource scheduling models are proposed for the single 
accident point and multiple accidents points, respectively. Finally, the genetic algorithms for solving the corresponding models are given.

\section{Emergency Rescue Scheduling Models}

The abstraction of the highway network is $G=\left(V, E, T, W_{(e, t)}, W_{(v, t)}\right)$, where $G$ denotes a directed weighted dynamic transport network, $V$ denotes a set of nodes in the highway network, $E$ denotes a set of directed segments connected to each node in the highway network, $T=\left[t_{0}, t_{m}\right]$ denotes the closed time interval beginning at time $t_{0}$ and ending at time $t_{m}$, while $W_{(e, t)}$ represents the set of time that vehicle $t$ waiting at node $v$ and $W_{(v, t)}$ represents the time required for the vehicle entering the road section $e$ at time $t$ to reach the section. In the actual traffic network, $T=\left\{t_{0}, t_{0}+\Delta t, t_{0}+2 \Delta t, \mathrm{~L}, t_{0}+M \Delta t, \mathrm{~L}, t_{m}\right\}$ where $\Delta t$ is the interval duration that is generally taken as 5 minutes. Without loss of generality, the change of road conditions within the interval duration is ignored.

The single-accident point (SAP for short) emergency rescue scheduling model can abstract as Sap $=(V, f, d, H)$, where $V$ represents highway network, $f$ in $V$ represents the node where the emergency vehicle is located, $d$ in $V$ represents the accident point. The objective of emergency rescue scheduling problem optimization can be described as: Emergency vehicle $i$ reaches the point of accident $j$ with minimum delay. Therefore, the objective function of the emergency rescue-scheduling problem is defined as:

$$
f_{\text {Sap }}^{*}=\min _{\forall \text { path: } v_{i} \rightarrow v_{j}} c_{i j}
$$

where $c_{i j}$ represents the delay of the emergency vehicle $i$ to the accident point $j$, including the road section travel time and the steering delay at the node. The entire route can be expressed as $v_{i} v_{i+1} \cdots$ $v_{m} v_{m+1} \cdots v_{j-1} v_{j}$. In order to ensure no loop, each node is different. $\mathrm{c}_{i j}$ can be defined by

$$
c_{i j}=\sum_{k=1}^{j-1} W_{e(k, k+1), t}+W_{v_{k}, t_{k}}
$$

with

$$
t_{k}=\left\{\begin{array}{l}
t_{k-1}+W_{e(k-1, k), t_{k-1}}+w_{v_{k}, t_{k-1}}, k=i+1, \mathrm{~L} j-1 ; \\
t_{0}, k=0 .
\end{array}\right.
$$

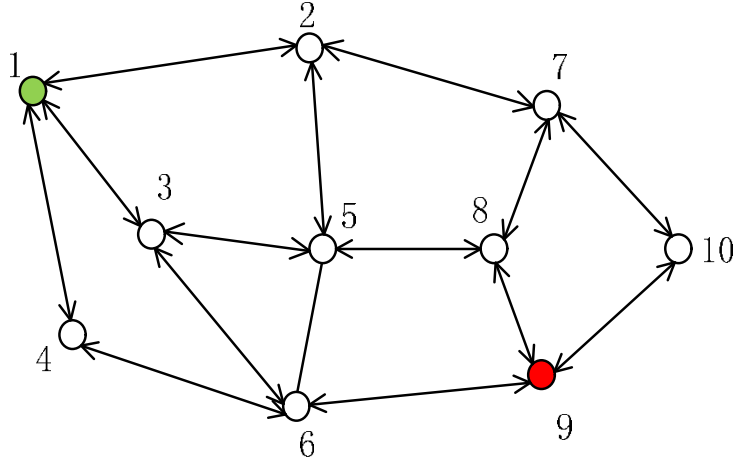

Fig.1 Single-Accident Point Emergency Rescue Scheduling
Table1 Road delay time

\begin{tabular}{|l|l|l|l|l|l|}
\hline & T0 & T1 & T2 & T3 & T4 \\
\hline $1<->2$ & $2 / 4$ & $3 / 2$ & $2 / 5$ & $4 / 4$ & $4 / 4$ \\
\hline $1<->3$ & $3 / 1$ & $2 / 1$ & $2 / 5$ & $5 / 4$ & $4 / 1$ \\
\hline $1<->4$ & $5 / 4$ & $5 / 1$ & $4 / 5$ & $1 / 1$ & $1 / 5$ \\
\hline $2<->5$ & $3 / 1$ & $3 / 2$ & $4 / 4$ & $5 / 3$ & $4 / 3$ \\
\hline $2<->7$ & $2 / 1$ & $4 / 1$ & $2 / 5$ & $3 / 4$ & $4 / 5$ \\
\hline $3<->5$ & $3 / 2$ & $2 / 3$ & $4 / 3$ & $3 / 2$ & $3 / 1$ \\
\hline $3<->6$ & $1 / 4$ & $4 / 5$ & $2 / 3$ & $1 / 2$ & $3 / 3$ \\
\hline $4<->6$ & $4 / 1$ & $2 / 2$ & $1 / 2$ & $1 / 3$ & $2 / 2$ \\
\hline $5<->8$ & $2 / 1$ & $4 / 2$ & $2 / 5$ & $5 / 5$ & $1 / 4$ \\
\hline $6<->9$ & $1 / 2$ & $5 / 2$ & $2 / 5$ & $4 / 4$ & $3 / 4$ \\
\hline $7<->8$ & $1 / 1$ & $3 / 1$ & $4 / 2$ & $2 / 3$ & $1 / 4$ \\
\hline $7<->10$ & $1 / 2$ & $3 / 1$ & $4 / 3$ & $4 / 5$ & $4 / 4$ \\
\hline $8<->9$ & $1 / 2$ & $5 / 4$ & $2 / 3$ & $1 / 1$ & $5 / 1$ \\
\hline $9<->10$ & $2 / 1$ & $5 / 4$ & $2 / 1$ & $5 / 1$ & $2 / 2$ \\
\hline
\end{tabular}

The Fig. 1 is used as an example for analysis. Assuming that the point where the vehicle is rescued is 1 , and the accident occurs at node 9, the delay on each road segment is a random value between $[1,5]$ (see Table 1 for specific values, such as the delay time of the $1 \leftarrow \rightarrow 2$ segment during 
the T_0 interval is $2 / 4$, where the numerator 2 represents a delay of $1 \rightarrow 2$, while the denominator 4 represents a delay of $2 \rightarrow 1$ ), and the stay time at each node is 0 .

We are looking for the best rescue path. For convenience, we use the symbol $T: a \rightarrow\{t\} b$ to indicate that during the time interval $\mathrm{T}$, a rescue vehicle takes $\mathrm{t}$ minutes to move from the point a to the point $b$. Then, 3 possible solutions are listed below:

$\leq$ Path 1:T_0:1 $\rightarrow\{3\} 3, T_{-} 0: 3 \rightarrow\{3\} 5, T_{-} 1: 5 \rightarrow\{4\} 8, T_{-} 3: 8 \rightarrow\{1\} 9$. That is, the total delay of Path 1 , along the nodes $1 \rightarrow 3 \rightarrow 5 \rightarrow 8 \rightarrow 9$, is 11 minutes.

$\leq$ Path 2: T_0:1 $\rightarrow\{5\} 4, T_{-} 1: 4 \rightarrow\{2\} 6, T_{-} 1: 6 \rightarrow\{5\} 9$. That is, the total delay of Path 2 , along the nodes $1 \rightarrow 4 \rightarrow 6 \rightarrow 9$, is 12 minutes.

$\leq$ Path 3:T_0:1 $\rightarrow\{2\} 2, T_{-} 0: 2 \rightarrow\{2\} 7, T_{-} 0: 7 \rightarrow\{1\} 8, T_{-} 2: 8 \rightarrow\{5\} 9$. That is, the total delay of Path 3 , along the nodes $1 \rightarrow 2 \rightarrow 7 \rightarrow 8 \rightarrow 9$, is 10 minutes.

Among them, we of course choose the best one, i.e. Path 3 along the nodes $1 \rightarrow 2 \rightarrow 7 \rightarrow 8 \rightarrow 9$.

\section{GA-based Solutions for the Proposed Model}

Although the optimization objective functions of the two emergency rescue models given in the previous section are relatively simple in form, in the calculation of the objective functions they all include one recursive process that is related to two types of weights $\mathrm{W}_{(\mathrm{e}, \mathrm{t})}$ and $\mathrm{W}_{(\mathrm{v}, \mathrm{t})}$. Therefore, the objective functions are difficult to describe with a simple linear or nonlinear function, and can only be calculated by a dynamic process. It is even difficult to determine whether the optimization model belongs to the category of convex programming. For such optimization problems, traditional optimization methods (such as conjugate gradient method, etc.) are ineffective. Considering the outstanding performance of genetic algorithm in solving many complex problems, this paper proposes to use genetic algorithm to solve the model.

The main difficulty for finding optimal solutions for the above problem lies in that the solution space is infinite, i.e., all possible paths over the given (directed) highway network $V$. Moreover, considering in practice, the conditions of road (such as traffic heavy, etc.) are time-variable. Thus, in this paper, we would like to use the concept of random walk to model a chromosome for the single-accident point rescue problem. More specifically, a chromosome is represented by a transition matrix for defining random walks (i.e. random solutions).

For the given Sap instance (1), the dimension of the transition matrix is defined by $|V|=n$. That is, when the start point $v_{i}$ and the objective point $v_{j}$ are specified, a chromosome $A=\left(a_{i j}\right)_{n \times n}$ is evaluated according to the following steps:

$\ddot{y} \quad$ Step 1: Get a random solution $P_{A}$ (i.e. a random walk path) from the rescue vehicle depot $v_{i}$ to the accident point $v_{j}$, according to the transition matrix $A$. That is, the probability of moving from the node $v$ to the node $v^{\prime}$ is $a_{v v^{\prime}}$.

$\ddot{y} \quad$ Step 2: Calculate the total delay of the rescue path $P_{A}$, denoted by $L_{A}$, according to the Eq.1,Eq.2 and Eq.3.

$\ddot{y} \quad$ Step 3: Repeat Step 1,2 for $m$ times, and calculate the average of $L_{A}$, denoted by $f_{\text {sap }}(A)$. That is, $f_{\text {sap }}(A)$ is the average of the total delay of rescue pathes that are induced by the transition matrix A.

$\ddot{y} \quad$ Step 4: Assign the fitness value of the chromosome $A$ as follow: $f(A)=1 /\left(1+f_{\text {sap }}(A)\right)$.

Note that, the above scaling technique is adopted considering that the objective is to minimize the total delay time for the rescue vehicle, instead of maximum.

After specified the chromosome coding method and fitness value evaluation strategy, we need to consider the crossover and mutation genetic operations. A valid chromosome must be a valid transition matrix, say, the total of each column/row should no large than 1 according the principle of probabilistic theory. Therefore, we introduce a new single-point crossover operation and two-point mutation operation as follows:

$\ddot{y}$ crossover: For the given two chromosomes (i.e. two transition matrices) $A$ and $B$, we sample $k$ in $[1: n]$ at random according the crossover probability $p_{c}$, then swap $k$-th row and $k$-th column of $A$ and $B$ simultaneously. 
$\ddot{y}$ mutation: For the given chromosome (i.e. a transition matrices) A, we sample $k_{1}, k_{2}$ in $[1: n]$ at random according the mutation probability $p_{m}$, then swap $k_{1}$-th row and $k_{2}$-th row, as well as $k_{1}$-th column and $k_{2}$-th column of A simultaneously.

Apparently, the above mutation operation is a self-crossover operation. This is simple but reasonable strategy since we use transition matrices as the space of valid chromosomes.

To proceed, we use the GA toolbox of MATLAB to solve the above model. The selection operation embedded in the toolbox is used without modification, while the crossover operation and mutation operation are given as above. We also need to write functions for evaluating the fitness values for a given chromosome $A$ according to Step 4. Of course, our coding work also includes a random walk algorithm for sampling random path according to the given transition matrix $A$, and then calculate the total delay according to Eq.1 , Eq. 2 and Eq. 3. Other aspects about our GA is listed below:

$\leq$ Population size: 30 ;

$\leq$ Maximal evolution generation: 100

$\leq$ Crossover probability: $p_{c}=0.75$

$\leq$ Mutation probability: $p_{m}=0.01$

$\leq$ Elite-keeping strategy: The best chromosome (i.e. with maximal fitness value) will be kept into the next generation without further mutation.

Our experiments are realized by using MATLAB 2016b, running at a notebook Lenovo ThinkPad with Intel Core i5-4300U CPU@1.9GHz and Window 8 OS. Towards the SAP instance given in the above section, our GA finds a better solution that is given as below: T_0:1 $\rightarrow\{3\} 3, T \_0: 3 \rightarrow\{1\} 6$, T_0:6 $\rightarrow\{1\} 9$. That is, the total delay of the above path, along the nodes $1 \rightarrow 3 \rightarrow 6 \rightarrow 9$, is 4 minutes.

\section{Further Discussions}

Finally, let us discuss the situation of multi-accident points (MAP for short). That is, there are multiple accidents happen almost simultaneously, and there are multiple rescue vehicles are ready for emergency rescue scheduling. Take the SAP model as a reference, it is not difficult to model the MAP problem as follows: $\operatorname{Map}=(V, F, D, H)$, where $V$ represents a highway network, $F$ represents a set of nodes where the emergency vehicles are located, $D$ represents a set of accident points, and $H$ represents the priority of each accident in $D$. The goal of optimizing the emergency rescue-scheduling problem can be described as: the total delay of the emergency vehicle reaching the accident site is the smallest.

With a newly introduced decisional $0-1$ variables $x_{i j}$ that is assigned 1 if the accident $j$ is arranged to be handled by the rescue vehicle $i$ and 0 otherwise, the objective function of the multi-accident emergency rescue scheduling problem is defined by

$$
f_{\text {Map }}^{*}=\min _{\substack{j \in D, i \in, x_{i, j} \in\{0,1\} \\ \forall p a t h: v_{i} \rightarrow v_{j}}} \sum h_{j} c_{i j} x_{i j}
$$

subject to

$$
\sum_{i \in F} x_{i j} \geq 1, \quad \forall j \in D
$$

where $\mathrm{c}_{\mathrm{ij}}$ is defined by the Eq. 2, and the constraint condition specified by Eq .5 indicates that each node in the accident point set $\mathrm{D}$ must dispatch emergency vehicles for rescue.

Furthermore, we employ the well-known Lagrange relaxation method [7] to combining the constraints into the objective function. Let $\lambda^{\prime}$ be a non-negative penalty vector corresponding to the constraint Eq.5. Then, the objective of the above model can be re-written as follows:

$$
\phi(X)=\min _{\substack{j \in D, i \in F, x_{i j} \in\{0,1\} \\ \forall p a t h: v_{i} \rightarrow v_{j}}} \sum h_{j} c_{i j} x_{i j}+<\lambda^{\prime}, \delta^{\prime}>
$$


where $\langle\cdot, \cdot\rangle$ is the inner-product of two $|V|$-dim vectors, while

$$
\delta^{\prime}(j)=1-\sum_{i \in F} x_{i j}, \quad j \in D
$$

Note that in the above formulation, $\lambda^{\prime}$ is in essential related to each vertex, while in practice, they should be updated according to certain strategy during the iteration process, and one can even to re-formulate $\lambda^{\prime}$ as a penalty functions that are related to the whole topology, instead of each vertex.

To solve the above MAP model, one can also use the GA-based method developed in this paper, with careful consideration the following differences

$\leq$ GA for the above MAP model can be organized into two layers: The first layer determines the optimal assignment on $x_{i j}(i$ in $D, j$ in $F)$, while the second layer determines optimal path for each rescue vehicle.

$\leq$ The chromosome consists two parts: the first part is a binary string for encoding $x_{i j}$, while the second part consists of $|D|$ transition matrices $A_{1}, A_{2}, \cdots, A_{|D|}$ for inducing random walk pathes for each rescue vehicle.

$\leq$ The standard single point crossover operation and multiple-point mutation operation embedded in the GA-toolbox of MATLAB can be directly used for $x_{i j}$ (i.e. the first part of chromosomes), while for $A_{1}, A_{2}, \cdots, A_{|D|}$ (i.e. the second part of chromosomes), the newly designed crossover and mutation operations introduced in the above section are recommended.

\section{Conclusions}

This paper presented an optimization model for emergency rescue scheduling problem in highway, and a novel genetic algorithm for solving the single-accident point model. Our GA is featured by taking random walk method and transition matrices as the chromosome encoding strategy. New crossover and mutation operations are also designed. Experiments suggest that our method is effective. In addition, the multiple-accident point case is also discussed in details.

\section{References}

[1] http://www.chinahighway.com/news/2017/1124559.php \} (Accessed on March 15, 2018.)

[2] Dong Yang, Lianyang Zhang, Yuzhu Zhang. The review on the current highway motor vehicle crashs in China. Medical Journal of Communications (in Chinese), 2016(5):443-446

[3] Tengjiao Fang. Research on Emergency Rescue Decision of Expressway[D], Chang'an University, 2017

[4] Xiaoshuai Dong, Zhengyuan Mao. The research on solution of dynamic VRP based on an improved genetic algorithm. Computer Engineering and Applications (in Chinese), 2018.

[5] Lei Fang, Jianmin He. Optimal location model and algorithm of emergency systems. Journal of systems engineering (in Chinese), 2003,18(1):49-54.

[6] K. Sundar and S. Rathinam. A prime-dual heuristic for a heterogeneous unmanned vehicle path planning problem. International Journal of Advanced Robotic Systems, Vol 2013(10):1-9, 2013.

[7] M.L. Fisher. The Lagrangian Relaxation Method for Solving Integer Programming Problems. INFORMS, 50(1):1-18, 2004. 